\title{
Rethinking 'did not attend': refocusing the spotlight on the child's needs
}

\author{
Kirby J, Harris J C. Development and evaluation of a 'was not brought' pathway: a team approach to managing children's missed dental appointments. \\ Br Dent J 2019; 227: 291-297 https://doi.org/10.1038/s41415-019-0621-z.
}

We intend to deliver care that is patient-centred, yet this is contradicted when children 'do not attend' (DNA) dental visits. Children do not take themselves to appointments; they have to be taken by parents or carers. Nonengagement with health services can be a sign of neglect, so missed dental appointments must be managed appropriately and consistently. With this view, the authors developed a new 'was not brought' (WNB) pathway for management of children and young people's (CYP) missed dental appointments that was implemented city-wide after piloting at a community dental service clinic.

The four stages of the WNB-CYP pathway provide a standardised, easy to follow system for contacting parents/carers (WNB1 - WNB3). WNB4 details further actions to follow multiple cancellations or lack of response to recall, including a risk of harm assessment and information sharing. Comprising an explanatory flowchart, templates for clinical notes with specific prompts for action, and editable template letters, the pathway had good overall compliance. Service evaluation over an eight-month pilot period found the pathway was used in $84 \%$ of occasions where children missed appointments. This led to contact being successfully made within three weeks and appointments rescheduled for $75 \%$ of WNBs managed using the pathway.

As well as improved consistency in management of missed appointments, reappointment in a timely manner, and acceptance by parents, there was a positive impact on staff. Semi-structured interviews highlighted how the pathway enabled and empowered every member of the whole skill-mixed dental team to contribute; nearly three-quarters of missed appointments were managed independently of dentist advice. Moreover, the pathway integrated well into existing systems and did not burden already busy workloads. Its introduction was actually perceived to improve efficiency and relieve professional uncertainty surrounding decision-making.

Stage WNB4 includes an information-sharing letter to the child's general medical practitioner. Pathway acceptability and staff engagement were further demonstrated by the dental team sharing information with other health and social care professionals beyond the express requirements of the pathway. However, the authors recognise the need for more direct and specific feedback regarding the value of information shared to maintain communication pathways.

Exchanging 'DNA' for 'WNB' shifts the focus from pressures on parents to the needs of the child. Having designed an effective approach to managing missed dental appointments that advocates for the child, the authors also plan to evaluate a version for vulnerable adults. Through engagement with the pathway, dental team members communicated a vision for change that could extend to other services. The importance of promoting child welfare and protecting vulnerable children is indisputable. But rather than rising to the level of our expectations, the reality is that we fall to the level of our systems. The WNB-CYP pathway weaves a tighter, more consistent net to ensure no vulnerable child slips through.

By Ji-Yun Stephanie Yeung

\section{Author Q\&A}

Jen Kirby

Leadership Fellow (Safeguarding Children) and Speciality Registrar in Paediatric

Dentistry, Health Education England Yorkshire and the Humber and Charles

Clifford Dental Services, Sheffield Teaching Hospitals NHS Foundation Trust, Sheffield, UK

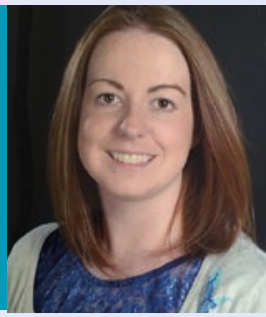

Why did you decide to undertake this study? For some years Sheffield community dental service had been trying to handle missed appointments better, recognising it as part of safeguarding children. Yet it was time-consuming and children were still slipping through the net. Tinkering around the edges of our protocols hadn't worked. So Jenny Harris developed and piloted a new 'was not brought' pathway for children and young people (WNB-CYP). We hoped it would help us be more consistent and that it would encourage us to share information earlier with other professionals. I came on board to evaluate whether it was working.

\section{Did any of the results surprise you?}

It was amazing to see how the whole team embraced change and took ownership as we rolled out the pathway to other clinics. We already knew we had a motivated team but until conducting the qualitative interviews I hadn't fully appreciated the extent to which staff engagement could drive positive change. The staff themselves were surprised how a simple change in terminology from 'did not attend' (DNA) to WNB inspired a dramatic change in their attitude. They focused more on putting the child's interests at the centre of their response. They also wanted to know how WNB would apply to vulnerable adults (adults at risk).

What do you think the next steps should be considering your findings?

This WNB-CYP green pathway has undoubtedly improved our practice and we continue to use it. We think this approach might bring similar benefits for other community dental services but it would also be good for colleagues to test it in other settings, such as dental practices and hospitals. We are currently asking doctors what they think of our information-sharing letters. We also intend soon to share a pink pathway for children subject to a child protection plan and are piloting a purple pathway for vulnerable adults. The ultimate test is whether this work actually helps safeguard children and young people from neglect. 